\title{
Reflets
}

Revue d'intervention sociale et communautaire

\section{Handicap et monde du travail : pratiques et perceptions}

\section{Sylvie Lirette, John Kabano, Charles Gaucher et Ann Beaton}

Volume 21, numéro 2, automne 2015

$20^{\mathrm{e}}$ anniversaire... déjà !

URI : https://id.erudit.org/iderudit/1035436ar

DOI : https://doi.org/10.7202/1035436ar

Aller au sommaire du numéro

Éditeur(s)

Reflets, Revue d'intervention sociale et communautaire

ISSN

1203-4576 (imprimé)

1712-8498 (numérique)

Découvrir la revue

Citer cet article

Lirette, S., Kabano, J., Gaucher, C. \& Beaton, A. (2015). Handicap et monde du travail : pratiques et perceptions. Reflets, 21(2), 131-160.

https://doi.org/10.7202/1035436ar

\section{Résumé de l'article}

Deux études ont été effectuées auprès de personnes ayant un handicap afin de cerner leur expérience par rapport au marché du travail. L'objectif de la première était de mieux saisir la perception du marché du travail qu'ont les personnes ayant un handicap. Des personnes ayant un handicap $(N=28)$, et qui sont à la recherche d'emploi, ont participé à des entrevues semi-dirigées. L'analyse effectuée permet de dégager la façon dont elles appréhendent l'accès à l'emploi. Dans un deuxième temps, des entrevues ont été menées auprès de personnes ayant un handicap visuel et occupant un emploi $(N=6)$. Celles-ci discutent du sens accordé à leur expérience sur le marché du travail. Les résultats sont abordés en fonction d'une vue d'ensemble des divers mouvements sociaux ayant contribué à l'évolution des droits en matière d'employabilité au Canada pour les personnes qui ont un handicap.
Tous droits réservés @ Reflets, Revue d’intervention sociale et communautaire, 2015
Ce document est protégé par la loi sur le droit d'auteur. L'utilisation des services d'Érudit (y compris la reproduction) est assujettie à sa politique d'utilisation que vous pouvez consulter en ligne. 


\section{Handicap et monde du travail : pratiques et perceptions}

Sylvie Lirette, M.A.

Université de Moncton

John Kabano, Ph. D.

Université du Québec à Rimouski

Charles Gaucher, Ph. D.

Université de Moncton

Ann Beaton, Ph. D.

Université de Moncton

\section{Résumé}

Deux études ont été effectuées auprès de personnes ayant un handicap afin de cerner leur expérience par rapport au marché du travail. L'objectif de la première était de mieux saisir la perception du marché du travail qu'ont les personnes ayant un handicap. Des personnes ayant un handicap $(N=28)$, et qui sont à la recherche d'emploi, ont participé à des entrevues semi-dirigées. L'analyse effectuée permet de dégager la façon dont elles appréhendent l'accès à l'emploi. Dans un deuxième temps, des entrevues ont été menées auprès de personnes ayant un handicap visuel et occupant un emploi $(N=6)$. Celles-ci discutent du sens accordé à leur expérience sur le marché du travail. Les résultats sont abordés en fonction d'une vue d'ensemble des divers mouvements sociaux ayant contribué à l'évolution des droits en matière d'employabilité au Canada pour les personnes qui ont un handicap.

Mots clés : Inclusion professionnelle, personne ayant un handicap, handicap et travail 


\section{Abstract}

Two studies were conducted to examine the work experience of people with disabilities. The purpose of the first study was to understand the perceptions that people with disabilities have of the labour market. The people with disabilities $(N=28)$, who participated in semi-directed interviews were all seeking employment. The analysis revealed how people with disabilities conceive of access to employment. At a second stage, interviews were conducted among participants with a visual impairment who were employed $(N=6)$. These employees discussed the meaning attributed to their experience in the labour market. Results will be addressed in light of various social movements that have contributed to the evolution of the right to work that is held by people with disabilities in Canada.

Key words: Professional inclusion, people with disabilitis, disability and work

\section{Introduction}

En 2006, l'Organisation des Nations Unies a adopté la nouvelle Convention relative aux droits des personnes ayant un handicap interdisant la discrimination fondée sur le handicap en matière d'emploi. L'ONU invite également les États à créer dans les principaux milieux de travail des possibilités pour les personnes qui ont un handicap et qui sont à la recherche d'un emploi (Organisation internationale du travail, 2006). Déjà depuis quelques décennies, le Canada met en place des mesures afin de faciliter l'inclusion professionnelle de ces personnes (Commission de la fonction publique du Canada, CFP, 2011). Malgré ces initiatives, leur participation sur le marché du travail a très peu changé au Canada (Ressources humaines et Développement des compétences Canada, 2008; Statistique Canada, 2014). Leur taux d'emploi se situe à $49 \%$ (Statistique Canada, 2014). Or, un grand nombre parmi elles veulent travailler et leur handicap ne 
les empêche pas de contribuer au marché de l'emploi (Panel on labour market, 2013). Cette sous-représentation et ce sousemploi de personnes ayant un handicap prévalent également au Nouveau-Brunswick. De fait, la province se situe parmi celles qui comptent le plus grand nombre de personnes ayant un handicap au Canada et leur présence sur le marché de l'emploi demeure inférieure à la moyenne nationale (Gouvernement du NouveauBrunswick, 2012).

Selon plusieurs études, la situation sur le marché du travail des personnes ayant un handicap est attribuable à la dynamique des rapports symboliques entre les agents sociaux impliqués (Deloitte, 2010; Fougeyrollas, et collab., 1998; Mercier, et collab., 1997). Ces personnes jugent de leur côté que les employeurs jouent un rôle déterminant dans leur embauche et leur maintien en emploi (Kabano, et Beaton, 2011). Or, les employeurs canadiens se disent mal préparés à leur inclusion professionnelle (Beaton, Kabano et Léger, 2012; Conseil canadien de la réadaptation et du travail, 2005). Pourtant, comme son titre l'indique, la Politique sur l'obligation de prendre des mesures d'adaptation pour les personnes handicapées dans la fonction publique fédérale (Secrétariat du Conseil du trésor du Canada, 2002) exige des employeurs une meilleure connaissance en matière d'inclusion en milieu de travail. Le présent article porte sur les défis de l'inclusion professionnelle des personnes ayant un handicap, et ce, selon leur perspective. Il sert à documenter deux dimensions de leur employabilité, soit leur accès à l'emploi et leur perception de leur inclusion professionnelle, et il s'appuie sur deux études menées à cet effet.

\section{Étude 1 : Les perceptions du marché du travail chez les personnes ayant un handicap}

Les personnes ayant un handicap ont une perception du monde du travail qui influe sur leur désir d'obtenir un emploi et sur leur motivation à le maintenir. Leur perception s'appuie à la fois sur leur vécu et sur les propos qu'elles entendent autour d'elles concernant le marché du travail. Elles se font une idée de 
"L'objectif de cette première étude était donc de mieux saisir la perception du marché du travail qu'ont les personnes ayant un handicap et qui sont à la recherche d'emploi."

\section{La méthode}

l'employé modèle et des dynamiques propres à un environnement de travail. L'objectif de cette première étude était donc de mieux saisir la perception du marché du travail qu'ont les personnes ayant un handicap et qui sont à la recherche d'emploi. Plus précisément, l'étude visait à 1 . définir la notion de marché du travail telle que perçue par les personnes ayant un handicap; 2. décrire le visage du marché du travail selon les personnes ayant un handicap et 3 . dresser un bilan des nouvelles approches d'intégration (éléments et stratégies) selon les personnes ayant un handicap.

\section{Le recrutement des participantes et des participants}

L'échantillon de l'étude était composé de personnes qui ont un handicap et qui, au moment de la collecte des données, désiraient travailler et se considéraient en mesure de le faire. Quoiqu'ils aient tous vécu une expérience préalable sur le marché du travail, aucun des participantes ou des participants n'occupait un emploi rémunéré au moment de l'étude. Ces personnes maintenaient un certain rapport avec le marché du travail et elles étaient prêtes à exprimer leurs perceptions de celui-ci et des enjeux qui influencent leur accès à l'emploi.

Leur recrutement a été effectué en collaboration avec des organismes de placement, comme l'Association du NouveauBrunswick pour l'intégration communautaire (ANBIC), avec des associations de personnes ayant un handicap, comme l'Association canadienne des paraplégiques, et avec le Centre pour la vie autonome de la Péninsule acadienne. Les participantes et les participants ont été ciblés en fonction des critères de diversifications suivants : la nature de leur handicap (moteur, visuel, auditif, cognitif, intellectuel ou lié à la santé mentale); le sexe ou le genre; l'âge; la scolarité. Deux régions au NouveauBrunswick ont été ciblées, soit le Grand Moncton et la Péninsule acadienne, lesquelles se distinguent par leur taux de chômage et par les services d'intégration en emploi mis à la disposition 
des personnes ayant un handicap. Le choix de ces deux régions permet une bonne vue d'ensemble en intégrant des contextes socio-économiques tout à fait différents.

Selon la procédure, les organismes ont sélectionné à partir de leurs bases de données les personnes qui répondaient aux critères de diversification, puis ont communiqué avec elles afin de leur présenter l'objectif de l'étude et les modalités de leur participation anonyme et confidentielle. Dans le cadre de ce contact, les organismes leur ont demandé l'autorisation de partager leurs coordonnées avec les chercheuses et chercheurs. Les organismes ont dressé une liste de personnes souhaitant participer à l'étude. Par la suite, les chercheuses et chercheurs ont communiqué par téléphone avec les participantes ou participants, revoyant avec eux les objectifs de l'étude, leur demandant s'ils souhaitaient toujours y participer, et, le cas échéant, leur fixant une rencontre. Toutes les entrevues ont été menées de personne à personne soit au domicile des participantes ou participants ou dans un endroit sûr et tranquille de leur choix. Quand une personne refusait de participer à l'étude, elle était systématiquement remplacée par une autre qui répondait aux mêmes critères de diversification.

\section{Les participantes et participants}

En tout, 28 personnes (14 femmes et 14 hommes) ayant un trouble de santé mentale $(n=5)$, un handicap auditif $(n=4)$, intellectuel $(n=5)$, moteur $(n=9)$ ou visuel $(n=5)$ ont partagé leurs visions du marché de l'emploi. Les participants et participants avaient complété l'école primaire $(10,7 \%)$, le secondaire $(42,9 \%)$ ou obtenu un diplôme d'un établissement postsecondaire (46,4\%).

"Les données recueillies à l'aide d'entrevues qualitatives ont permis de mettre en relief le positionnement des personnes ayant un handicap face au marché du travail." La majorité d'entre eux étaient âgés de 34 ans ou moins (67\%). Toutes ces personnes étaient sans emploi au moment des entrevues. Elles habitaient dans la région de la Péninsule acadienne $(n=$ 14) ou dans la région du Grand Moncton $(n=14)$. Les données recueillies à l'aide d'entrevues qualitatives ont permis de mettre en relief le positionnement des personnes ayant un handicap face au marché du travail. Notons que tous les participantes et participants étaient francophones et que $46 \%$ de l'échantillon était constitué de personnes ayant un handicap depuis la naissance. 


\section{Les mesures}

La grille d'entrevue regroupe des questions permettant de saisir les perceptions du marché du travail (images, expressions et normes), le portrait de l'employé modèle ou de la candidate ou du candidat idéal et les approches d'intégration, sujets tels qu'abordés par les participantes ou participants à l'étude. Ces thèmes ont permis de souligner quelques pistes de solution pour stimuler et améliorer l'inclusion sur le marché du travail des personnes qui ont un handicap.

Dans la cueillette de données se rapportant à la connaissance du monde du travail, la question la plus importante porte sur les perceptions des personnes. À cet égard, nous leur posons une question ouverte d'évocation libre, sans contrainte et multiple, qui permet d'identifier les images qu'elles y associent : À quoi vous fait penser le marché du travail pour les personnes ayant un handicap?

Sur le plan analytique, l'évocation libre a l'avantage de fournir d'emblée les unités d'enregistrement dont il reste à trouver l'organisation. Des questions supplémentaires permettent de contrer tout biais de lecture des mots évoqués - biais souvent liés à leur polysémie - d'en préciser le sens, et de respecter le plus possible la pensée des sujets; le tout sert à établir des catégories exclusives et exhaustives. L'ensemble des réponses à ces questions constitue une source importante d'expression de perceptions.

Enfin, au moyen de questions portant sur le thème de la candidate ou du candidat idéal à l'emploi, la participante ou le participant est amené à évoquer son propre rapport au marché du travail. Dans une certaine mesure, ce que la personne perçoit de la candidate ou du candidat idéal est le reflet des préférences qu'elle reconnait chez l'employeur et sur lesquelles elle s'appuie indirectement pour évaluer sa propre compétence face au monde du travail. De plus, la participante ou le participant est appelé à identifier les facteurs qui favorisent l'embauche des personnes ayant un handicap, et ceux qui lui nuisent. Enfin, les participantes et participants sont invités à identifier les conditions d'interventions destinées à promouvoir l'insertion professionnelle des personnes 
ayant un handicap. Les réponses à ces questions constituent un corpus idéal pour l'élaboration de stratégies favorisant l'insertion professionnelle. La durée des entrevues est de 45 minutes ou plus, selon le nombre d'évocations libres venant de la participante ou du participant et selon sa capacité de développer son propos.

\section{Le plan d'analyse}

Le traitement du matériau discursif a été réalisé à l'aide de l'analyse de contenu (Paillé, et Mucchielli, 2003) et au moyen de catégories induites. Les chercheuses et chercheurs ne disposaient pas de catégories préétablies avant la collecte des données, mais une analyse du contenu utilisant un système de catégorisation leur a permis d'en dégager des catégories. Cette approche a fait en sorte que le traitement des données demeure fidèle au langage des participantes et des participants, à leur vocabulaire et à l'organisation qu'ils accordent à leurs discours.

La procédure d'analyse a suivi les étapes de base suivantes de l'analyse de contenu (Mucchielli, 1991) :1. la lecture du matériel; 2. la formation des catégories, leur analyse et leur comparaison; 3. leur regroupement dans des concepts plus larges et plus englobants. Selon le modèle retenu pour l'entrevue (non directif, combiné aux associations libres), l'unité d'analyse porte sur les mots qui ont été évoqués spontanément. Ce sont ces derniers qui ont fait l'objet de catégorisations et qui ont servi à dégager le rapport au marché du travail qu'entretiennent les personnes ayant un handicap. En bref, il s'agissait de découvrir les noyaux structurants des perceptions. Le logiciel d'analyse informatisé Nvivo a servi au traitement de données discursives.

Réalisée d'abord individuellement par les chercheuses et chercheurs, l'analyse de contenu a permis d'identifier un certain nombre de catégories induites. Par la suite, ces derniers se sont rencontrés afin de comparer leurs systèmes respectifs de catégorisation en vue d'une structuration commune des discours des répondants. Lors d'échanges (par téléphone ou internet) consécutifs à cette rencontre, certains de ces systèmes ont été remaniés et de nouveaux ont pu être créés. Ce travail commun 
sur la catégorisation a abouti à un système de catégories conforme aux données recueillies.

\section{Les résultats et la discussion}

Les analyses ont permis de définir la notion du marché du travail, la place que réserve le marché du travail aux personnes ayant un handicap, la candidate ou le candidat idéal recherché par les employeurs ainsi que des approches d'intégration.

\section{Le marché du travail}

"Les personnes ayant un handicap ont des images du marché du travail constituées d'une variété d'opinions, de préjugés et de stéréotypes. Leur environnement social, leur famille, l'école, les médias leur ont transmis des informations qui modèlent leurs perceptions, leur vision particulière du marché du travail et de la place qu'elles pourraient ou ne pourraient pas $y$ occuper. »
Les personnes ayant un handicap ont des images du marché du travail constituées d'une variété d'opinions, de préjugés et de stéréotypes. Leur environnement social, leur famille, l'école, les médias leur ont transmis des informations qui modèlent leurs perceptions, leur vision particulière du marché du travail et de la place qu'elles pourraient ou ne pourraient pas y occuper.

Qu'est-ce que le marché du travail : perception générale

Lors de l'étude, les participantes ou participants ont utilisé divers mots et expressions pour caractériser le marché du travail. Cela a permis d'en dresser le portrait d'ensemble, tel qu'esquissé par les personnes qui ont un handicap. Ainsi, "marché du travail " se définit comme un lieu où s'effectue un travail en particulier, telle une garderie, ou comme un travail spécifique, tel être machiniste ou relieur; tandis que l'« emploi » consiste en une activité agréable et adéquate qui convient à la personne; celle-ci retire du plaisir des fruits de son travail. Selon les participantes ou participants, travailler est une question de passion :la personne employée aime ce qu'elle fait et elle est motivée par son emploi. Dans un marché du travail qui semble offrir diverses possibilités, cette dernière peut alors choisir un emploi comportant des tâches qu'elle aimerait accomplir et qui la motiveraient considérablement. Elle n'est plus limitée ou restreinte à un type d'emploi en particulier. Si elle le désire, elle peut s'épanouir sur le marché du travail grâce à une variété de milieux de travail et de types d'emploi. Cette perception n'est toutefois pas sans soulever d'interrogations lorsque l'on constate les multiples échecs d'insertion que connaissent plusieurs 
"Cette perception n'est toutefois

pas sans soulever

d'interrogations

lorsque l'on constate les multiples échecs d'insertion que connaissent plusieurs groupes de personnes, dont celles vivant avec un handicap."

"Cette perception de la "rigide flexibilité " du marché du travail rejoint ce qu'évoque Castel dans Les métamorphoses $d u$ salariat (1995), à savoir que l'apparente souplesse du marché du travail couve de nouvelles contraintes qui imposent toujours davantage à l'employé la responsabilité de conserver son emploi.» groupes de personnes, dont celles vivant avec un handicap. Comme le rappelle Merzouk (2008), ces dernières sont confrontées à des obstacles importants dans leur désir d'accéder au marché du travail.

Les participantes ou participants reconnaissent que le marché du travail est soumis à des normes spécifiques de productivité, d'efficacité, de rendement et d'organisation. Même si le secteur de l'emploi s'est diversifié au sein de notre société postindustrielle, les travailleuses et les travailleurs se voient confrontés à certaines règles spécifiques.

«Comme sérieux, c'est quand-ce que t'es là pour rentrer. Si tu travailles 8 à 8 le soir, t'es là pour travailler. T'es pas là pour compter des jokes... Pis workaholic, y'a du monde qui travaille qui se lève le matin qui travaille qui vont se coucher. Pis ponctuel, c'est montrer comment tu prends le travail au sérieux si tu es ponctuel.» (Gabriel, problème de santé mentale)

Occuper un emploi c'est se soumettre à une routine bien établie, à des tâches déterminées et à un horaire fixe de travail, et ce, en dépit du fait que le marché du travail est devenu plus flexible et permet aux employés de travailler à forfait, à temps partiel ou de manière occasionnelle. De plus, même si le monde du travail est devenu plus inclusif pour les personnes ayant un handicap, tout n'est pas permis. Certains règlements ayant trait au temps et à l'organisation d'un emploi s'appliquent à tous. Et lors de l'entrevue d'embauche, la candidate ou le candidat doit prouver qu'il est capable de satisfaire à des normes de fonctionnement auxquelles nul employé ne saurait se soustraire : disponibilité, efficacité, productivité et respect des horaires. Pour les participantes ou participants, le respect des horaires de travail est un indicateur du sérieux investi dans le travail et de la passion pour celui-ci. Cette perception de la "rigide flexibilité » du marché du travail rejoint ce qu'évoque Castel dans Les métamorphoses du salariat (1995), à savoir que l'apparente souplesse du marché du travail couve de nouvelles contraintes qui imposent toujours davantage à l'employé la responsabilité de conserver son emploi. 
"Moi, quand je

cherche un travail, je pense que je suis capable. Parce que je me connais, je connais mes capacités, je sais ce que je peux faire et ce que je ne peux pas faire. Mais, un employeur quand il me voit, quand il remarque que je suis un handicapé, il pense que je suis incapable. »
Pour les participantes ou participants, être sur le marché du travail signifie également avoir des patrons, côtoyer d'autres employés, servir des clientes ou des clients et utiliser de l'équipement divers. Même si la société a fait en sorte que les conditions de travail se sont individualisées au fil du temps, ils croient qu'occuper un emploi signifie automatiquement être en contact direct avec d'autres gens, soit des collègues toujours présents, un superviseur auquel il faut se rapporter et répondre de ses actes et aussi des clientes ou clients qu'il faut satisfaire. La vision du travail décrite par les participantes ou participants n'inclut pas le travail à son propre compte, ce qui est par ailleurs révélateur du peu d'écho qu'a reçu le mouvement deVie autonome auprès des populations francophones du Canada. Et pour eux, l'essence même d'un travail consiste à être rémunéré et à respecter certaines contraintes liées à son organisation : productivité, efficacité, rythme de travail et horaires.

\section{La place des personnes ayant un handicap sur le marché du travail}

Malgré une certaine ouverture du marché du travail envers les personnes qui ont un handicap, les participantes ou participants croient qu'il est parsemé d'obstacles résultant de la précarité des emplois qui leur sont réservés et des salaires de misère qui leur sont versés. Il y a un manque d'emplois qui offrent des salaires convenables leur permettant de payer leurs factures. Les personnes doivent déployer beaucoup d'efforts pour décrocher un emploi et c'est peine perdue dans la majorité des cas.

«Les employeurs n'ont pas la même vision que moi que toute autre personne qui est handicapée. Moi, quand je cherche un travail,je pense que je suis capable. Parce que je me connais, je connais mes capacités, je sais ce que je peux faire et ce que je ne peux pas faire. Mais, un employeur quand il me voit, quand il remarque que je suis un handicapé, il pense que je suis incapable. » (Gilles, handicap moteur) 
On remarque ici que les participantes ou participants ont tout d'abord décrit le marché du travail pour l'ensemble de la population. Lorsqu'ils en viennent à leurs situations de travailleuses ou de travailleurs ayant un handicap, leur perception differe. L'image du monde du travail s'assombrit et n'est plus la même :

«Une personne vraiment autonome, c'est facile pour lui de se trouver de l'emploi, c'est facile. Lui qui a besoin de se prouver c'est difficile, ça devient rare... Où est-ce qu'on va le placer? " (William, handicap visuel)

La recherche d'un emploi ou l'idée d'entrer sur le marché du travail fait peur aux personnes qui ont un handicap. Elles s'inquiètent de ce qui va leur arriver, de la façon dont les autres vont les traiter et les accueillir. Elles s'interrogent sur leurs capacités d'effectuer le travail adéquatement.

"C'est épeurant parce que quand t'entends pas, c'est difficile d'aller sur le marché du travail, parce que moi j'me sens, à part tout le temps bien sûr,j'trouve ça gênant...J'trouve ça peurable. J'suis vraiment pas préparée à ça. " (Mélanie, handicap auditif)

Ce manque de confiance en leurs capacités au moment d'entrer sur le marché du travail fait en sorte qu'elles perçoivent déjà leur travail comme étant difficile et qu'elles pensent qu'elles ne gagneront pas assez d'argent pour subvenir à leurs besoins. Elles anticipent ainsi ce que travailler pourrait être avant même de tenter leur chance. Elles doutent et se questionnent. Pourtant, au cours des années 1970, le mouvement de défense et de promotion des droits pour les personnes ayant un handicap a lutté de façon intensive afin qu'elles aient les mêmes droits que tout autre citoyen, incluant celui d'occuper un emploi. Comme mentionné plus haut, divers programmes et lois ont été développés afin que ces personnes ne soient plus victimes de discrimination au travail en raison de leur handicap. Malgré ces acquis, elles redoutent encore une entrée sur le marché du travail. De plus, les dynamiques 
"...les participantes

ou participants ont soulevé le fait que celles qui ont un handicap intellectuel sont beaucoup plus stigmatisées, suivies de celles qui ont des difficultés sur le plan du langage.» sociales sur le marché du travail représentent pour elles un fardeau. Elles perçoivent comme étant très difficiles les rapports sociaux sur les lieux de travail. Elles sont même convaincues que les interactions avec les autres employés et même avec l'employeur seront tendues.

«S'ils ne comprennent pas ton problème pis ils ne savent pas pourquoi t'es malade, ce monde-là $\mathrm{y}$ te regardons pis y disons y veut pas travailler. Ils en a qui peuvent dire ça pis y comprennent pas trop bien là. » (Mélanie, handicap auditif)

Si de manière générale les personnes ayant un handicap sont victimes de discrimination sur le marché du travail, les participantes ou participants croient que certaines parmi elles font l'objet d'une plus grande stigmatisation que les autres. La discrimination ne frapperait donc pas de la même manière toutes les personnes qui ont un handicap. À titre d'exemple, les participantes ou participants ont soulevé le fait que celles qui ont un handicap intellectuel sont beaucoup plus stigmatisées, suivies de celles qui ont des difficultés sur le plan du langage.

"Ben a peut pas s'expliquer comme il faut, quand on parle de travailler, mais elle ne peut pas expliquer comme il faut quoisse qu'est son potentiel à elle. D'après moi elle ne peut pas se vendre, elle ne peut pas se mettre en valeur. " (Mélanie, handicap auditif)

Il existerait donc toujours une certaine discrimination fondée sur le type de handicap. Les propos émis laissent croire que les personnes ayant un handicap visuel ou sur le plan moteur seraient moins discriminées et auraient le plus de facilité à être embauchées.

\section{La candidate ou le candidat idéal}

En plus d'avoir une image ou une vision précise du monde du travail, les participantes ou participants à l'étude ont également discuté de leurs perceptions de l'employé idéal, soit la personne dont l'employeur est constamment à la recherche. Il s'agit d'une 
personne respectueuse, pas trop gênée, capable de communiquer avec les autres, et qui répond aux normes de fonctionnement du marché du travail tout en se montrant sociable envers les autres employés et l'employeur. L'employé idéal suit les politiques et les procédures propres à l'emploi décroché et contribue à l'avancement de l'entreprise grâce à son attitude et à ses habiletés.

«Une personne qui est toujours à temps, qui est très prête toujours à faire quelque chose que l'on demande, ben toujours faire oui... toujours prêt à le faire... » (Philip, handicap visuel)

Toujours selon les participantes ou participants, l'employé idéal interagit efficacement avec les membres d'une équipe de travail et est doté d'une aptitude à communiquer avec les autres. Il témoigne du respect envers son employeur.

«Il faut que tu le respectes le plus possible... tu vas avoir certaines tâches à faire, il faut que tu les respectes, faut que tu les fasses. Si ton boss te dit faut que c'est faite en deux jours, ben faut que c'est faite en deux jours. » (Marco, handicap moteur)

Le respect, les habiletés dans le domaine de la communication et les capacités d'interagir avec autrui seraient alors des champs dans lesquels les employés doivent exceller. Quant aux employeurs,

"Quant aux employeurs, les participantes ou participants croient qu'ils recherchent un employé capable de travailler, peu importe les conditions d'emploi telles que le revenu, la charge de travail, la pression au travail et les exigences des horaires de travail. " les participantes ou participants croient qu'ils recherchent un employé capable de travailler, peu importe les conditions d'emploi telles que le revenu, la charge de travail, la pression au travail et les exigences des horaires de travail.

"Pis même si t'aimes pas ça ou si t'aimes ça... $\mathrm{y}$ faut t'es prêt à grandir la compagnie... pis tu ne peux pas avoir une attitude négative contre ton travail parce que ça se démontre.» (Gabriel, problème de santé mentale)

«Il faut que t'es dévoué à $100 \%$, si possible $110 \%$ comme qu'on dit quand on travaille. Faire toutes les choses le meilleur possible, le meilleur. » (Marco, handicap moteur) 
Pour les participantes ou participants, la candidate ou le candidat idéal posséderait une attitude positive et serait motivé et enthousiaste. Il serait fiable, il ferait preuve d'honnêteté, il serait soigné de sa personne et n'aurait aucun handicap. D'après les participantes ou participants, même à compétence et expérience de travail égales, les employeurs choisiraient toujours la candidate ou le candidat qui n'a pas de déficience. La candidate ou le candidat idéal fait preuve de compétences professionnelles et intellectuelles adaptées aux exigences du marché du travail : formation adéquate, bilinguisme, aptitude à prendre des décisions, débrouillardise, polyvalence et expérience antérieure de travail.

Les personnes interrogées pourraient selon elles obtenir un emploi comme tout autre candidate ou candidat idéal si l'employeur ne tenait pas compte des facteurs liés à l'intégrité physique, intellectuelle ou psychique, bref, à leur handicap. En adoptant un tel point de vue, elles renoncent à se voir comme candidates idéales et, se disant inaptes à remplir l'ensemble des critères, elles ne croient plus en leurs chances d'être choisies.

«Une fois que tu sens que tu as toute qu'est-ce qui veulent, moi, j'trouve ça difficile avec un handicap, parce qu'on dirait que les employeurs recherchent quelqu'un qui en a pas un.» (Marie, handicap visuel)

Les personnes qui ont un handicap ne doivent pas se montrer exigeantes envers les employeurs; elles se doivent d'accepter les conditions de leur emploi. Ainsi, les participantes et les participants ne semblent croire en aucun accommodement ou négociation qui pourrait faciliter leur entrée dans le monde du travail.

"Si l'employeur pense que ça va lui coûter de l'argent supplémentaire, je peux t'assurer qu'ils ne vont pas t'embaucher. Ils croient aussi qu'il ne doit pas avoir de "fautes" chez l'employé. Cette image idéalise le type d'employé recherché et présente les employeurs comme sans reproche et recherchant la perfection. " (David, handicap auditif) 


\section{Les approches d'intégration}

Les participantes ou participants à l'étude ont aussi discuté de conditions de travail qualifiées d'acceptables et de création d'emplois susceptibles de promouvoir leur intégration au monde du travail et de les amener à se réaliser pleinement. Grâce à une évaluation approfondie de ses habiletés, une personne ayant un handicap pourrait dans le cadre d'un emploi remplir des tâches adaptées à ses capacités. Les participantes ou participants pensent également que le travail doit contribuer au mieux-être des individus. Il procure à la personne des éléments essentiels à l'estime de soi, au sentiment d'autoaccomplissement, à la fierté et à la confiance en soi. Il doit procurer de meilleures perspectives d'avenir et favoriser un meilleur état mental et physique. Des conditions de travail saines pourraient même atténuer les effets sociaux, physiques et psychologiques (isolement, stigmatisation, discrimination) engendrés par le handicap. Un jumelage adéquat entre l'emploi décroché, les aptitudes et les intérêts de la personne ayant un handicap est donc essentiel à son épanouissement en tant qu'employée. Selon les participantes ou participants, le travail permet d'être plus heureux et autonome. Il valorise l'individu ayant un handicap et lui offre la chance d'atteindre certains objectifs, tel celui de fonder une famille. Notons que

"...même s'ils ont décrit le marché du travail comme leur étant plutôt hostile, les participantes ou participants lui reconnaissent des effets positifs à long terme sur leur qualité de vie. » même s'ils ont décrit le marché du travail comme leur étant plutôt hostile, les participantes ou participants lui reconnaissent des effets positifs à long terme sur leur qualité de vie. Occuper un emploi leur permettrait de répondre de manière autonome à leurs besoins immédiats (se nourrir, se vêtir, etc.) et leur estime d'eux-mêmes s'en trouverait améliorée. De plus, en occupant un emploi, les personnes ayant un handicap se voient faisant partie d'une communauté d'appartenance (Galarneau, et Radulescu, 2009). Castel (1995) affirme que travailler permet de créer des liens sociaux et de tisser un réseau social autour de soi. Dans le cas des personnes ayant un handicap, cela signifie surtout atténuer l'isolement qu'elles vivent en demeurant exclues du monde du travail ou de la société en général. 
"Les participantes ou participants rapportent également le rôle important que doivent jouer les agents de placement en tant qu'intermédiaires. »
Les participantes ou participants ont également discuté du besoin criant de ressources visant à faciliter leur recherche d'un emploi. Ils pourraient en effet bénéficier d'encadrement pour déterminer le type d'emploi qui leur convient, de conseils sur les techniques d'entrevue, d'information sur l'équipement spécialisé, de préparation au moment d'intégrer le monde du travail et de soutien tout au long de leur recherche d'un emploi ou en cours d'emploi.

«Donc j'ai fait face à la discrimination beaucoup puis j'trouve que quand j'ai arrivé ici avec [nom de l'organisme d'aide à l'emploi] eux autres m'ont dit que si j'avais eu cette expérience-là que ça ne veut pas dire que ça va arriver de nouveau... Pis ça j'apprécie ça parce que ça me fait vouloir chercher encore plus pour de l'emploi.» (Marie, handicap visuel)

Les participantes ou participants rapportent également le rôle important que doivent jouer les agents de placement en tant qu'intermédiaires. Ils doivent être bien présents et faciliter les contacts entre l'employé ayant un handicap et leur futur employeur. Cela rappelle très bien l'importance du rôle que jouent souvent les agences externes, notamment l'Institut national du Canada pour les aveugles (INCA) ou le Conseil canadien de la réadaptation et du travail (CCRT) impliquées actuellement dans l'inclusion professionnelle des personnes ayant un handicap.

En somme, selon les résultats de cette recherche, nous constatons que les personnes qui ont un handicap s'inscrivent en opposition aux normes de fonctionnement du marché du travail. Le manque de flexibilité constaté chez ce dernier, les salaires de misère qui le caractérisent et les discriminations perçues laissent voir que la personne ayant un handicap ne se sentirait pas la bienvenue. Le fait que les participantes ou participants admettent que l'employeur recherche la candidate ou le candidat idéal et le fait qu'ils se croient inaptes à satisfaire ses attentes démontrent un certain antagonisme entretenu envers le marché du travail. Notons que les résultats révèlent également des nuances quant 
à la discrimination interhandicap. Parmi les candidates ou candidats à l'embauche, ce sont les personnes aqui ont un handicap moteur ou visuel qui ont plus de facilité à décrocher un emploi. À point nommé, la deuxième des études exposées dans le présent article porte sur le vécu de six personnes ayant un handicap visuel au moment d'intégrer le marché de l'emploi dans la région du Grand Moncton.

\section{Étude 2 : Présentation d'une expérience spécifique d'intégration au travail de personnes ayant un handicap visuel}

Cette étude avait pour but de mieux comprendre le vécu de personnes ayant un handicap visuel et de saisir leurs perceptions et interprétations de leurs expériences au moment d'intégrer le marché du travail.

\section{La méthode}

Le recrutement des participantes et des participants

Le recrutement des participantes ou participants s'est effectué en collaboration avec le bureau de Moncton du CCRT et le Conseil du premier ministre sur la condition des personnes ayant un handicap, dont le bureau est situé à Fredericton. Un échantillonnage théorique a été réalisé en fonction de caractéristiques qui se sont précisées au fur et à mesure de l'évolution de la recherche (Strauss, et Corbin, 1998), et ce, à partir des particularités suivantes : la personne sélectionnée devait être francophone ou anglophone; être âgée de 19 à 65 ans; avoir un handicap visuel et occuper un emploi ou avoir occupé un emploi dans trois régions du Grand Moncton au Nouveau-Brunswick : Dieppe, Riverview ou Moncton. Toutes les entrevues se sont déroulées de personne à personne, dans un endroit paisible. 


\section{Les participantes et participants}

"Les participantes ou participants à l'étude étaient complètement avengles ou avaient une faible vision, c'està-dire qu'ils étaient considérés comme légalement aveugles et dans l'impossibilité de détenir un permis de conduire. »
Les participantes ou participants à l'étude étaient complètement aveugles ou avaient une faible vision, c'est-à-dire qu'ils étaient considérés comme légalement aveugles et dans l'impossibilité de détenir un permis de conduire. L'échantillon était constitué de trois femmes et de trois hommes, dont cinq occupaient un emploi à temps plein dans différents domaines, dont l'administration, la vente et les ressources humaines. Un participant était à la retraite. Deux des cinq participantes ou participants encore en emploi faisaient du bénévolat. Les participantes ou participants ont occupé un emploi pour une durée de dix ans en moyenne; ils ont occupé chacun trois emplois différents depuis leur entrée sur le marché du travail. Un participant avait complété le secondaire tandis que les autres avaient obtenu une éducation postsecondaire. Parmi les six participantes et participants, trois avaient effectué un retour aux études après avoir obtenu un premier diplôme postsecondaire et avoir occupé un emploi pendant au moins trois ans. Au moment de l'étude, ces trois participantes ou participants avaient terminé leurs études et occupaient un poste.

\section{Les mesures}

Ces personnes ont participé à une entrevue semi-dirigée avec questions ouvertes, ce qui a permis d'explorer leurs perceptions en profondeur à partir de cinq questions qui abordaient les thèmes suivants :

1. les défis auxquels les personnes ayant un handicap visuel sont confrontées au moment d'entrer sur le marché du travail (par exemple, l'adaptation du milieu de travail à leur situation, le choix d'un transport, un nouveau style de vie et les décisions à prendre pour occuper un emploi);

2. les ressources qu'elles utilisent pour réussir sur le marché du travail (par exemple, les organismes communautaires et les programmes gouvernementaux);

3. les sentiments qu'elles éprouvent tout au long de leur expérience d'accès au marché du travail (par exemple, la frustration, l'isolement, la fatigue, la satisfaction et l'étonnement); 
4. les apprentissages découlant de leur expérience de travail (par exemple, l'affirmation de soi, la connaissance de ses besoins et de ses limites et la confiance en soi); et

5. le sens que ces personnes donnent à leur expérience sur le marché du travail en observant les facteurs de réussite, la vitesse de travail limitée ou l'effet de la chance. Les entrevues ont duré une heure en moyenne.

\section{Le plan d'analyse}

Une analyse de contenu thématique, dont le principe réside dans la construction de catégories possédant chacune un sens propre (Mayer, et collab., 2000), a été réalisée à partir des propos des participantes et des participants. L'analyse de contenu permet en effet de dégager la signification du discours pour ensuite en dégager les éléments principaux. Il est par la suite possible de décrire et de comparer le contenu des propos tenus (Mayer, et

"L'analyse de contenu a permis de cerner certaines tensions qui marquent l'expérience des personnes interviewées à leur entrée sur le marché du travail et qui mènent aux deux constats principaux développés dans les prochains paragraphes: un environnement non adapté... et le fait que chaque travailleuse ou travailleur avec handicap visuel est responsable de sa propre intégration au travail." collab., 2000). Les thèmes de l'analyse ont été tirés de la grille semi-structurée qui a servi à réaliser les entrevues réalisées auprès des personnes.Ainsi, pour procéder à l'analyse des données, chacun des verbatims d'entrevue a été catégorisé en fonction des questions contenues dans le canevas d'entrevue. Les propos ont par la suite été comparés, ce qui a permis de dégager quelques constats qui se rapportent aux conditions de travail des personnes ayant un handicap visuel et qui ont été regroupés selon des thèmes qui leur étaient communs.

\section{Les résultats et la discussion}

L'analyse de contenu a permis de cerner certaines tensions qui marquent l'expérience des personnes interviewées à leur entrée sur le marché du travail et qui mènent aux deux constats principaux développés dans les prochains paragraphes : un environnement non adapté (qui fait en sorte que pour réussir, les personnes doivent posséder certaines aptitudes non reliées à leur profession au moment d'entrer sur le marché du travail) et le fait que chaque travailleuse ou travailleur avec handicap visuel est responsable de sa propre intégration au travail. Les défis et les stratégies reliés à ces deux principaux constats sont abordés par la suite. 
"Malgré différentes mesures législatives mises en place au Canada au cours des cinquante dernières années pour en assurer l'accès, beaucoup de milieux de travail n'offrent pas systématiquement de moyens d'adaptation à leurs employés qui ont un handicap."
Le milieu de travail : environnement hostile ou seulement indifférent aux personnes ayant un handicap visuel?

Malgré différentes mesures législatives mises en place au Canada au cours des cinquante dernières années pour en assurer l'accès, beaucoup de milieux de travail n'offrent pas systématiquement de moyens d'adaptation à leurs employés qui ont un handicap. Au moment de leur embauche et pour accomplir efficacement leurs tâches, ils doivent souvent pourvoir leurs propres mesures d'adaptation, ces dernières étant limitées, voire inexistantes. Plusieurs témoignages recueillis dans le cadre de cette étude ont permis de documenter ce manque d'adaptation a priori des milieux de travail. Comme en témoigne cette participante, les outils de base pour accomplir son travail n'ont été adaptés qu'après sont entrée en fonction, et ce, malgré le fait que ses besoins étaient somme toute très faciles à combler : un écran de 21 pouces et le logiciel Zoomtext :

"Puis l'INCA a embarqué dedans aussi. So, ils ont plus débattu le cas que moi pour avoir un écran de 21 pouces. Puis à la fin, l'employeur a payé pour l'écran." (Karine)

D'autre part, de façon moins évidente cette fois-ci, l'environnement physique de cette même employée causait également un problème :l'éclairage devait être modifié en raison de sa sensibilité à la lumière. Or, ces adaptations n'ont été apportées que dans les semaines qui ont suivi son entrée en fonction et non au moment de l'embauche. Par ailleurs, certains participants ou participantes ont dû se procurer eux-mêmes le logiciel JAWS de lecture d'écran afin de travailler de façon autonome à l'ordinateur.

"J'ai dû faire des appels afin de trouver une compagnie de distribution et me procurer moimême le logiciel Jaws pour pouvoir travailler de façon indépendante sur mon ordinateur. J'ai dû attendre quelques semaines avant de le recevoir et pouvoir utiliser mon ordinateur au bureau.» (Marc) 
"Afin d'être autonomes, les participantes ou participants ont $d \hat{u}$ entreprendre euxmêmes la tâche d'adapter leur milieu de travail. Leurs témoignages portent à croire que les employeurs ne répondent pas de manière systématique à leur obligation." "
Encore une fois, ces besoins spécifiques, pourtant essentiels à l'accomplissement des tâches de chacune et de chacun de ces employés avec handicap visuel, n'ont été comblés qu'après leur entrée en poste, ce qui a pu retarder ou compromettre les apprentissages de base initiaux à tout nouvel emploi.

Afin d'être autonomes, les participantes ou participants ont dû entreprendre eux-mêmes la tâche d'adapter leur milieu de travail. Leurs témoignages portent à croire que les employeurs ne répondent pas de manière systématique à leur obligation. Comme l'explique un participant, il a dû avoir recours au CCRT pour payer les frais de la mise à jour des logiciels nécessaires à son travail.

"J'ai fait toutes les démarches pour obtenir des mises à jour des logiciels que j'utilise. L'employeur a payé après coup, mais en plus de mes tâches au bureau, je devais faire celles-là aussi. J'ai dû appeler quelques compagnies pour obtenir les meilleurs prix.» (André)

Dans le cas d'une participante, l'employeur a défrayé le coût de l'achat d'un écran de 21 pouces, mais seulement après que le CCRT et l'INCA soient intervenus afin de lui expliquer ses responsabilités envers une employée ayant un handicap visuel. Ces exemples démontrent encore une fois à quel point les employeurs ne semblent pas connaître leurs responsabilités envers leurs employés qui ont un handicap, mais surtout que le réflexe d'adaptation de l'environnement de travail est loin d'être acquis et repose bien souvent sur les épaules de ces derniers.

Un autre élément contextuel très important contribue à cette situation : les technologies spécialisées nécessaires à l'adaptation des milieux de travail sont peu disponibles dans la province du Nouveau-Brunswick. Comme le mentionnent certains participantes ou participants, étant donné le nombre restreint de citoyennes ou citoyens qui ont à l'utiliser, cet équipement spécialisé doit venir d'ailleurs au pays, et même des États-Unis. Des frais de manutention et d'envoi s'ajoutent alors à son prix de vente. Une fois les logiciels et les pièces d'équipement reçus, leur installation se fait par l'acheteur avec l'aide de son entourage. 
En cas de difficultés en cours d'installation, de l'aide peut être obtenue par téléphone. Étant donné la spécificité de tels logiciels et pièces d'équipement, plusieurs ont mentionné que les techniciens réguliers des entreprises ou des compagnies informatiques locales peuvent très difficilement leur prêter assistance quant à leur installation ou à leur utilisation. Ainsi, les personnes qui doivent utiliser ces nouvelles technologies sont laissées à ellesmêmes. Elles doivent faire appel aux tutoriels ou modes d'emploi de leurs nouveaux produits que les compagnies diffusent sur Internet (par exemple, Freedom scientific, www.freedomscientific. $\mathrm{com} /$ training). Cet apprentissage prend un certain temps, ce qui peut ralentir le rythme de travail du nouvel employé qui a un handicap. Comme l'ont rapporté deux des personnes interviewées, la personne nouvellement embauchée peut se montrer frustrée et impatiente, et se sentir isolée et épuisée à cause du processus. En plus de se familiariser avec les nouvelles tâches à accomplir, elle doit apprendre par ses propres moyens à utiliser de l'équipement spécialisé afin de travailler de façon efficace et autonome. Voilà qui ajoute une étape supplémentaire et constitue un réel défi au moment de commencer un nouvel emploi.

L'environnement de travail ne s'arrête toutefois pas au poste de travail; il inclut aussi un quartier, une localité, une paroisse ou une ville en particulier, de même que des infrastructures qui ne conviennent pas toujours aux personnes qui ont un handicap. Au Nouveau-Brunswick, faute de planification, de considération ou de mise en œuvre d'une accessibilité universelle, ces personnes ont généralement de la difficulté à avoir accès à tous les services et installations qui sont à la portée de l'ensemble de la population (Conseil du premier ministre sur la condition des personnes ayant un handicap, 2010). Pourtant, le Code du bâtiment du Nouveau-Brunswick met actuellement à la disposition des travailleuses et travailleurs de la construction des normes visant à améliorer l'accessibilité des lieux publics (Conseil du premier ministre sur la condition des personnes ayant un handicap, 2010). Par exemple, un participant qui prenait l'autobus pour se rendre au travail déplorait l'absence de trottoirs dans cette zone urbaine, ce qui rendait cet environnement non sécuritaire pour lui. La 
question de l'accessibilité physique du milieu de travail pour les personnes ayant un handicap dépasse donc la nécessité de responsabiliser les employeurs : elle implique une transformation sociétale majeure qui engage les autorités dans un processus de conception universelle des lieux publics et de l'aménagement du territoire en général.

\section{L'individualisation des conditions d'inclusion sur le marché du travail}

Les participantes ou participants mentionnent avoir pu identifier eux-mêmes leurs besoins, mais ils ont dû recourir aussi à une aide externe pour connaitre la technologie requise leur permettant d'être autonomes dans leur environnement de travail. Certains précisent avoir été guidés par un employé de l'INCA afin d'obtenir les logiciels et pièces d'équipement qui leur permettaient d'adapter adéquatement leurs postes de travail respectifs. Un autre a eu recours aux services d'un agent du CCRT afin d'obtenir le financement nécessaire à la mise à jour de certains logiciels. Les expériences de ces participantes ou participants démontrent bien qu'il y a eu au fil des ans une extension perverse de la notion d'autonomie. La personne ayant un handicap visuel doit entreprendre elle-même les démarches auprès d'organismes autres que l'entreprise pour obtenir des conseils et du financement lui permettant d'adapter à ses besoins son milieu de travail. En bref, la responsabilité lui revient de s'assurer que tout est en place pour qu'elle puisse accomplir efficacement son travail.

Cette individualisation des conditions de travail va plus loin.

"...la personne qui a un handicap et qui a choisi d'entrer sur le marché du travail pour subvenir à ses propres besoins doit souvent s'imposer d'importants compromis et prendre des décisions difficiles..."
Dans le contexte de non-reconnaissance de la nécessité d'une conception universelle de l'aménagement déjà abordé, la personne qui a un handicap et qui a choisi d'entrer sur le marché du travail pour subvenir à ses propres besoins doit souvent s'imposer d'importants compromis et prendre des décisions difficiles, contrairement aux personnes qui n'ont pas de handicap. Un participant de l'étude a dû quitter son milieu rural pour habiter en ville afin de bénéficier du transport en commun. Il s'est aussi acheté une maison près d'un arrêt d'autobus dans un quartier de la ville qu'il n'aurait pas nécessairement choisi. Pour la même 
"Force est donc de constater à nouveau qu'il incombe à la personne qui a un handicap de gérer les situations de travail, que ce soit en intervenant auprès des employeurs, des collègues, de la clientèle, ou par tout autre moyen, incluant l'humour." raison, une participante a vendu sa maison à la campagne, où elle habitait depuis une vingtaine d'années, pour emménager dans un appartement du centre-ville. Dans le cadre de son emploi, un participant doit surmonter la difficulté de se rendre et de participer à des évènements publics. Il doit donc planifier ses déplacements longtemps à l'avance et s'assurer d'avoir quelqu'un pour l'y conduire. Il explique également avoir eu à enregistrer l'assurance du véhicule au nom de son épouse afin qu'elle puisse le conduire à certains évènements. Cette dernière a aussi dû modifier son propre horaire pour pouvoir le conduire chaque jour au travail. Pour une autre participante, c'est une voisine qui l'aide et la conduit au travail pendant la saison estivale, période pendant laquelle le service de transport en commun est interrompu dans sa région.

Enfin, un autre aspect illustrant l'individualisation des conditions d'accès au marché du travail réside dans les diverses tâches supplémentaires que doivent accomplir les personnes ayant un handicap afin d'être autonomes dans un nouvel emploi. D'après les témoignages reçus, la personne ayant un handicap visuel doit posséder toute une gamme d'habiletés et d'aptitudes qui ne sont pas en lien avec les compétences requises pour accomplir le travail pour lequel elle a été embauchée. On s'attend qu'elle soit en mesure d'adapter de façon adéquate son poste de travail, de se familiariser rapidement avec son nouvel environnement et, concurremment, de se familiariser avec de nouvelles tâches. De telles attentes nécessitent beaucoup de confiance en soi, d'affirmation de soi, une connaissance approfondie de ses besoins et de ses limites ainsi qu'un excellent sens de la planification et de l'organisation. Un participant souligne également qu'il faut être bien dans sa peau et à l'aise avec son problème visuel afin d'en discuter ouvertement avec ses collègues de travail et son employeur. Il revient ainsi à l'employé de rassurer son patron et ses collègues quant aux implications de son handicap sur les tâches à accomplir. Enfin, un participant explique comment il a recours à son sens de l'humour pour rendre certaines situations moins tendues avec ses clients et ses collègues de travail. Force est donc de constater à nouveau qu'il incombe à la personne qui a un handicap de gérer les situations de travail, que ce soit en 
intervenant auprès des employeurs, des collègues, de la clientèle, ou par tout autre moyen, incluant l'humour.

Cette situation qui implique une constante surcharge pour la personne ayant un handicap et nouvellement employée n'est pas étrangère au manque de soutien collectif qui caractérise notre époque postprovidentielle. Dans le domaine du handicap, au nom de l'autonomie, on confie de plus en plus à cette personne non seulement la tâche d'identifier ses besoins en matière d'emploi et d'y répondre, mais aussi celle de trouver elle-même les ressources, et même de les payer. Plus encore, la personne qui a un handicap est responsable de trouver les façons de se rendre par elle-même au travail et d'en fréquenter les lieux. Pour s'inscrire dans la dynamique de son emploi, elle doit enfin faire preuve d'aptitudes particulières, qui vont bien au-delà des compétences requises pour accomplir ses tâches régulières.

\section{Discussion}

«Face aux

bouleversements

sociétaux qui ont

marqué le XX siècle,

l'inclusion sur le

marché du travail

de la personne qui

a un handicap a graduellement changé

de visage. "
Face aux bouleversements sociétaux qui ont marqué le $\mathrm{XX}^{\mathrm{e}}$ siècle, l'inclusion sur le marché du travail de la personne qui a un handicap a graduellement changé de visage. "Comment rendre cette personne productive? " semble s'être métamorphosé en "Comment faire en sorte que cette personne occupe un emploi? ». Au fil des années 1970 et 1980, grâce à diverses initiatives mises de l'avant par des groupes de pression et des dirigeantes ou des dirigeants, les personnes ayant un handicap ont acquis les mêmes droits que tous les autres citoyennes ou citoyens. Désormais, il est interdit de discriminer ces personnes en raison de leur handicap et elles peuvent ainsi occuper un emploi sans porter au quotidien le fardeau de discriminations systémiques. De plus, des programmes gouvernementaux encouragent fortement les employeurs à embaucher des personnes qui ont un handicap. Cependant, comme en font foi les deux études présentées dans cet article, le marché de l'emploi comporte encore des défis pour ces dernières, notamment ceux reliés à l'accessibilité des environnements de travail, à la perception que les personnes 
concernées se font du marché du travail et à l'individualisation des conditions d'inclusion au moment d'y accéder. Les mesures d'adaptation systémiques devant être mises en place par les employeurs sont encore aujourd'hui insuffisantes.

Les deux études soulignent l'importance de sensibiliser les employeurs aux réalités de la personne qui tente de se tailler une place dans un milieu de travail. Au dire des personnes ayant un handicap, les attitudes des employeurs correspondent au facteur le plus déterminant dans leur accès à l'emploi (Fraser, et collab., 2011). Or, des recherches révèlent que ces derniers entretiennent des préjugés à leur endroit (Beaton, Kabano et Léger, 2012). Ils sont d'avis qu'elles seront incapables d'un rendement conforme aux normes du marché de l'emploi, qu'elles exigeront davantage des congés ainsi que des aménagements et, par conséquent, qu'elles entraîneront pour l'entreprise des coûts supplémentaires (Harcourt, Lam et Harcourt, 2005). Ce sont des perceptions erronées (Vornholt, Uitdewilligen et Nijhuis, 2014). Par exemple, les résultats d'un sondage d'envergure mené auprès des employeurs révèlent que le coût moyen de l'aménagement des personnes ayant un handicap est de moins de $500 \$$ (Job Accommodation Network, 2014).

Les deux études montrent par ailleurs des expériences tout à fait différentes des personnes ayant un handicap. Les participantes ou participants de la première étude ont fait état de préjugés et de discrimination à leur endroit. En revanche, celles et ceux de la deuxième étude ont moins parlé de discrimination, mettant plutôt l'accent sur l'effort à déployer pour s'intégrer au marché du travail et y connaître du succès, et s'interrogent sur les attitudes à adopter ou sur les stratégies et les actions à entreprendre pour percer en tant que personnes ayant un handicap. Se pourrait-il dès lors que les personnes qui ont un handicap soient peu préparées pour une entrée sur le marché du travail et le perçoivent avec plus d'angoisse? D'ailleurs, plusieurs d'entre elles ne savent pas quelles attitudes adopter devant les défis qu'elles doivent soudainement surmonter. De plus, certaines hésitent à foncer et à démontrer de l'affirmation de soi et une grande initiative en raison de fausses 
"Les politiques

d'inclusion des

personnes ayant un

handicap ne suffisent

pas pour produire des

changements; il faut

aller au-delà de la

loi et proposer aux

gens un fondement

rationnel à toute

démarche visant

l'inclusion." croyances et idées préconçues entretenues sur le marché du travail pour les personnes ayant un handicap. La crainte de faire face à de la discrimination au travail les freine dans leurs élans.

Les politiques d'inclusion des personnes ayant un handicap ne suffisent pas pour produire des changements; il faut aller au-delà de la loi et proposer aux gens un fondement rationnel à toute démarche visant l'inclusion. Une piste prometteuse s'inspire du modèle de la transformation des représentations sociales (Flament, 1994). Selon leurs éléments structuraux, ce sont les dimensions périphériques plutôt que le noyau central qui sont les plus susceptibles de transformation. Cette périphérie s'adapte à un contexte particulier " en maintenant la signification centrale et l'intégration de l'information nouvelle sans faire apparaitre un trop gros bouleversement " (Abric, 1994, p. 76). Ainsi, les personnes qui ont un handicap et les intervenantes ou intervenants des organismes d'aide collaboreraient à développer des stratégies visant à changer les mentalités des employeurs réticents à leur embauche ou à leur maintien en emploi. Par exemple, selon les principes du modèle de la transformation des représentations sociales, si un employeur refuse d'embaucher une personne ayant un handicap à cause des coûts des aménagements, non seulement il faut lui montrer qu'il peut en assumer les coûts, mais surtout que les aménagements contribueront au développement de son entreprise en facilitant son accès à plusieurs autres personnes. Tout changement se veut important; l'individu ne change pas ses habitudes pour le seul plaisir de le faire; il faut une justification à tout changement, un fondement rationnel. Si l'employeur est rassuré sur le fait que les aménagements demandés ne vont pas nuire à sa clientèle régulière, mais possiblement l'élargir, il va peut-être les accepter d'emblée. Dans tous les cas, il faut lui donner des exemples tangibles et mettre l'accent sur les avantages immédiats du changement. En somme, le modèle de transformation des représentations sociales permettrait aux intervenantes, aux intervenants et aux personnes ayant un handicap de mieux cerner les valeurs et les sentiments des employeurs et de justifier le changement auquel ils convient ces derniers. À ce jour, aucune étude n'a examiné l'effet d'une approche axée sur la transformation des représentations sociales 
"Afin de réduire les obstacles à l'emploi, certains auteurs soulignent l'importance de préparer la nouvelle génération des employeurs et des intervenantes ou intervenants sociaux aux défis de l'inclusion professionnelle des personnes ayant un handicap dans le cadre d'une économie mondiale du savoir en pleine mouvance..." appliquée aux pratiques des employeurs en matière d'inclusion des personnes ayant un handicap.

Le présent article sert à sa façon à pallier un manque d'ouvrages portant sur les perceptions des personnes ayant un handicap par rapport au marché de l'emploi. Mais les deux études qui y sont décrites comportent des limites qui se doivent d'être mentionnées. D'abord, les réalités des personnes ayant un handicap varient énormément selon leur sexe et leurs limitations (Ren, Paetzold et Colella, 2008), éléments dont les deux études ne tiennent pas compte, ce qui a pour résultat de présenter une perspective limitée des enjeux associés à leur inclusion sur le marché du travail. Notons également la taille restreinte des échantillons des études et le fait que les participantes ou participants habitent tous au Nouveau-Brunswick, ce qui ne met en lumière qu'une partie de la réalité entourant l'inclusion de ces personnes au marché de l'emploi au Canada.

Cette recherche s'inscrit dans la foulée du mouvement de promotion de la participation des personnes ayant un handicap au marché de l'emploi. Afin de réduire les obstacles à l'emploi, certains auteurs soulignent l'importance de préparer la nouvelle génération des employeurs et des intervenantes ou intervenants sociaux aux défis de l'inclusion professionnelle des personnes ayant un handicap dans le cadre d'une économie mondiale du savoir en pleine mouvance (Gilbride, et collab., 2003; Stensrud, 2007). Dans cette perspective, la recherche devrait servir à outiller les employeurs et les intervenantes ou intervenants de demain et les sensibiliser à l'employabilité des personnes qui vivent avec un handicap.

\section{Bibliographie}

ABRIC, Jean-Claude (1994). «Les représentations sociales : aspects théoriques », dans Jean-Claude ABRIC (dir.), Pratiques sociales et représentations, Paris, Presses universitaires de France, p. 11-35.

BEATON, Ann M., John KABANO et Mélanie LÉGER (2012). "How do employers view people with diabilities in the labour market? ", Revue Développement humain, handicap et changement social, Vol. 20, p. 25-39.

CASTEL, Robert (1995). Les métamorphoses de la question sociale. Une chronique du salariat, Paris, Fayard, 490 p. 
COMMISSION DE LA FONCTION PUBLIQUE DU CANADA (2011). «Historique de l'équité en matière d'emploi dans la fonction publique — Le rôle de la Commission de la fonction publique du Canada », réf. du 8 juin 2015, http://www.psc-cfp.gc.ca/plcy-pltq/eead-eeed/rprt/ ee-psc-cfp/index-fra.htm\#toc1.2

CONSEIL CANADIEN DE LA RÉADAPTATION ET DU TRAVAIL (2005). " Final report Diversity planning for inclusive employment ", réf. du 15 septembre 2011, http://www.ccrw. org/userfiles/CCRW-DPIE-EN.pdf

CONSEIL DU PREMIER MINISTRE SUR LA CONDITION DES PERSONNES HANDICAPÉES (2010). "Semaine de sensibilisation de la situation des personnes handicapées ", réf. du 15 septembre 2012, http://www.gnb.ca/0048/index-f.asp

DELOITTE (2010). «Pour une plus grande inclusion : L'intégration des personnes handicapées en milieu de travail. Sommaire des tables rondes, Parlons de diversité ", réf. du 15 septembre 2011, http://www.senseability.ca/docs/default-source/articles/articles-fr/deloitte-pour-une-plusgrande-inclusion.pdf?sfvrsn $=4$

FLAMENT, Claude (1994). "Aspects périphériques des représentations sociales ", dans Christian GUIMELLI (dir.), Structures et transformation des représentations sociales, Neufchâtel (Suisse), Delachaux et Niestlé, p. 85-118.

FOUGEYROLLAS, Patrick, et collab. (1998). « Processus de production du handicap - Classification québécoise. Québec : Réseau international sur le processus de production du handicap ", réf. du 15 septembre 2011, http://www.ripph.qc.ca/mdh-pph/comment-utiliser-mdh-pph/ classification-quebecoise-processus-production-du

FRASER, Robert, et collab. (2011). «Understanding employers' hiring intentions in relation to qualified workers with disabilities: Preliminary findings " Journal of Occupational Rehabilitation, Vol. 20, p. 420-426.

GALARNEAU, Diane, et Marian RADULESCU (2009). "L'emploi chez les personnes ayant un handicap ", réf. du 6 octobre 2013, http://www.statcan.gc.ca/pub/75-001-x/2009105/ article/10865-fra.htm

GILBRIDE, Denis, et collab. (2003). «Identification of the characteristics of work environments and employers open to hiring and accommodating people with disabilities ", Rehabilitation Counseling Bulletin,Vol. 46, p. 130-137.

GOUVERNEMENT DU NOUVEAU-BRUNSWICK (2012). "Plan d'action-emploi pour les personnes ayant un handicap au Nouveau-Brunswick », réf. du 8 juin 2015, http://www2.gnb. $\mathrm{ca} /$ content/dam/gnb/Departments/pcsdp-cpmcph/pdf/publications/EAPReport2012FR.pdf

HARCOURT, Mark, Helen LAM, et Sondra HARCOURT (2005). « Discriminatory practices in hiring: Institutional and rational economic perspectives ", The International Journal of Human Resource Management,Vol. 16, p.2113-2132.

JOB ACCOMMODATION NETWORK. (2014). «Workplace accommodations: Low cost, high impact ", réf du 8 juin 2015, https://askjan.org/media/downloads/LowCostHighImpact.pdf

KABANO, John, et Ann M. BEATON. (2011). «Le marché du travail vu par des personnes ayant un handicap : une étude de leurs représentations sociales ", Revue Développement humain, handicap et changement social,Vol. 19, 109-126.

MAYER, Robert, et collab. (2000). Méthodes de recherche en intervention sociale, Éditeur Gaetan Morin. $410 \mathrm{p}$. 
MERCIER, Michel, et collab. (1997). « Approche des représentations sociales relatives à l'emploi des personnes handicapées en Région wallonne : Rapport de la recherche effectuée à la demande du Ministre W.Taminiaux, Ministre de l'Action sociale, du Logement et de la Santé ", Belgique, Facultés universitaires Notre-Dame de la Paix. réf du 15 septembre 2011, https://pure.fundp.ac.be/ws/files/969075/38617.pdf

MERZOUK, Rachid (2008). " Travail, handicap et discrimination: lorsque le travail devient aussi un espace de production du handicap ", Reflets : revue d'intervention sociale et communautaire, Vol. 14, p. 155-181.

ORGANISATION INTERNATIONALE DU TRAVAIL (2006). "L'OIT salue la nouvelle convention de l'ONU sur les droits des personnes handicapées ", réf. Du 7 octobre 2012, http://www.ilo.org/global/about-the-ilo/press-and-media-centre/news/WCMS_080633/ lang--fr/index.htm

PANEL ON THE LABOUR MARKET OPPORTUNITIES FOR PEOPLE WITH DISABILITIES (Panel) (2013). "Rethinking disability in the private sector», Ottawa, ON: Public Works and Government Services of Ontario ", réf. du 8 juin 2015, http://digitalcommons.ilr.cornell.edu/cgi/viewcontent.cgi?article=1564\&context $=$ gladnetco llect

REN, Lily Run, Ramona L. PAETZOLD et Adrienne COLELLA (2008). "A meta-analysis of experimental studies on the effects of disability on human resource judgments ", Human Resource Management Review,Vol. 18, p. 191-203.

RESSOURCES HUMAINES ET DÉVELOPPEMENT DES COMPÉTENCES CANADA (2008). «Rapport fédéral de 2008 sur les personnes handicapées :Vers l'intégration des personnes handicapées ", No. de cat. ISSD-001-12-08F. réf. du 8 juin 2015,

http://www.rhdcchrsdc.gc.ca/fra/condition_personnes_handicapees/rapports/rhf/2008/ rhf_2008.pdf

SECRÉTARIAT DU CONSEIL DUTRÉSOR DU CANADA (2002). «Politique sur l'obligation de prendre des mesures d'adaptation pour les personnes handicapées dans la fonction publique fédérale », réf. du 8 juin 2015, http://www.tbs-sct.gc.ca/pol/doc-fra.aspx?id=12541

STATISTIQUE CANADA (2014). "Persons with disability and employment », Catalogue no. 75006X, Ottawa, Gouvernement du Canada, 1-15. réf. du 8 juin 2015, http://www.statcan.gc.ca/ pub/75-006-x/2014001/article/14115-eng.pdf.

STENSRUD, Robert. (2007). "Developing relationships with employers means considering the competitive business environment and the risks it produces ", Rehabilitation Counseling Bulletin, Vol. 50, p. 226-237.

STRAUSS, Anselm, et Juliet CORBIN (1998). Basics of qualitative research techniques and procedures for developing grounded theory, (2 $2^{\mathrm{e}}$ edition), Londre, Sage, $400 \mathrm{p}$.

VORNHOLT, Katharina, Sjir UITDEWILLIGEN et Frans J.N. NIJHUIS (2014). « Factors affecting the acceptance of people with disabilities at work: A literature review ", Journal of Occupational Rehabilitation, Vol. 23, p. 463-475. 